\title{
Using a Passive Fiber Ring Cavity to Generate Shot-Noise-Limited Laser Light for Low-Power Quantum Optics Applications
}

\author{
Malcolm B. Gray, Jong H. Chow, Kirk McKenzie, and David E. McClelland
}

\begin{abstract}
High-stability single-frequency fiber lasers typically exhibit relative intensity noise that is far in excess of shot noise across a broad frequency range extending from dc to many tens of megahertz. This excess noise precludes the use of these fiber lasers in a range of precision interferometry and quantum optics applications. We present experimental results using a passive, high $Q$, fiber ring resonator to filter out up to $26 \mathrm{~dB}$ of excess laser intensity noise, resulting in a stabilized laser output that reaches the shot-noise limit over most of its signal spectrum.
\end{abstract}

Index Terms-Laser noise suppression, passive fiber resonator, passive fiber ring cavity (FRC).

\section{INTRODUCTION}

$\mathbf{M}$ ANY precision optics experiments require that laser intensity noise approaches the limits set by the quantum mechanical nature of light: the fundamental shot-noise limit. These include gravitational wave (GW) detection, which employs the Pound-Drever-Hall (PDH) tecnhiqe [1], optical parametric amplifiers used to generate squeezed states of light [2], and other quantum optics experiments.

Similarly, a shot-noise-limited source is also required in continuous variable quantum cryptography, where there is intense topical interest and activity for systems related to secure communications. In this application, the signal beam must be shotnoise-limited across the entire channel bandwidth, as it is the quantum mechanical noise which enables perfect security of the system [3]. To date, most of the quantum cryptography experiments employ solid-state Nd:YAG lasers, whose superior noise performance enables experimental proof-of-concept. For practical cryptographic systems, on the other hand, sources that operate in a telecommunications window, such as in the $1550-\mathrm{nm}$ wavelength range, would be preferred for long-distance communications.

At the 1550-nm wavelength range, however, commercially available free running semiconductor and fiber lasers do not meet these requirements. To exemplify this problem, we have plotted the laser intensity noise of a single frequency, low noise distributed Bragg-reflector (DBR) fiber laser in Fig. 1, Trace A, where the measurement was made for a laser power of $900 \mu \mathrm{W}$.

Manuscript received October 9, 2006; revised March 4, 2007. This work was supported by the Australian Research Council (ARC) under the auspices of the Australian Consortium for Interferometric Gravitational Astronomy.

The authors are with the Centre for Gravitational Physics, Faculty of Science, The Australian National University, Canberra, ACT 0200, Australia.

Color versions of one or more of the figures in this letter are available online at http://ieeexplore.ieee.org.

Digital Object Identifier 10.1109/LPT.2007.899466

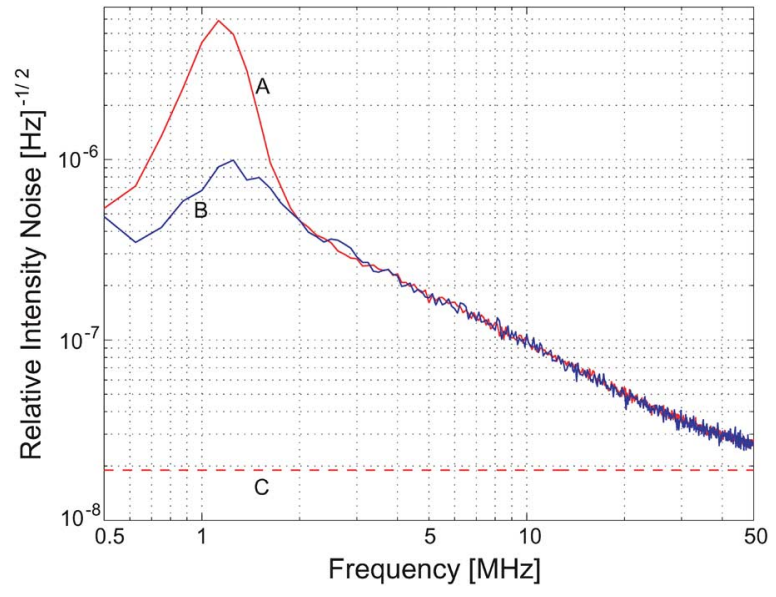

Fig. 1. (A) Free running relative intensity noise for our DBR fiber laser. (B) Relative intensity noise of the same laser with the RIN control circuit activated. (C) Shot-noise-limited RIN produced by the detection of $900 \mu \mathrm{W}$ of light on a detector with a responsivity of $\rho=1 \mathrm{~A} / \mathrm{W}$ at a wavelength of $1550 \mathrm{~nm}$.

The shot-noise-limited relative intensity noise (RIN) of an optical measurement is given by

$$
\mathrm{RIN}_{\text {shotnoise }}=\sqrt{\frac{2 e}{\eta P_{\mathrm{opt}}}}
$$

where $e$ is the electronic charge, $\eta$ is the photodetector responsivity in amperes per watt, and $P_{\text {opt }}$ is the optical power detected. At $900 \mu \mathrm{W}$, this yields a shot-noise-limited RIN of $1.8 \times$ $10^{-8} / \sqrt{\mathrm{Hz}}$, shown as Trace C. The laser relaxation oscillation (RO) in Trace A at $\sim 1.2 \mathrm{MHz}$ can be seen to be approximately $50 \mathrm{~dB}$ above the shot noise. In addition to the RO, we also observe broadband noise extending from $1.2 \mathrm{MHz}$ to more than $50 \mathrm{MHz}$. This broadband noise has a spectral density that scales approximately as the inverse of signal frequency, and approaches the shot-noise limit at frequencies above $50 \mathrm{MHz}$.

The most common method to reduce the laser RIN is by active stabilization of the pump diode. Fig. 1, Trace B, shows the laser intensity noise with the in-built active RIN controller system switched ON. This system samples the laser output and provides feedback to the pump diode, thereby stabilizing the laser intensity to suppress the RO by more than $15 \mathrm{~dB}$. However, it is evident that the associated broadband noise is unaffected and still extends to frequencies above $50 \mathrm{MHz}$.

Due to the inherent time delays associated with photodetection, electronic amplification and feedback actuation, the practical bandwidth of active laser stabilization systems is limited 


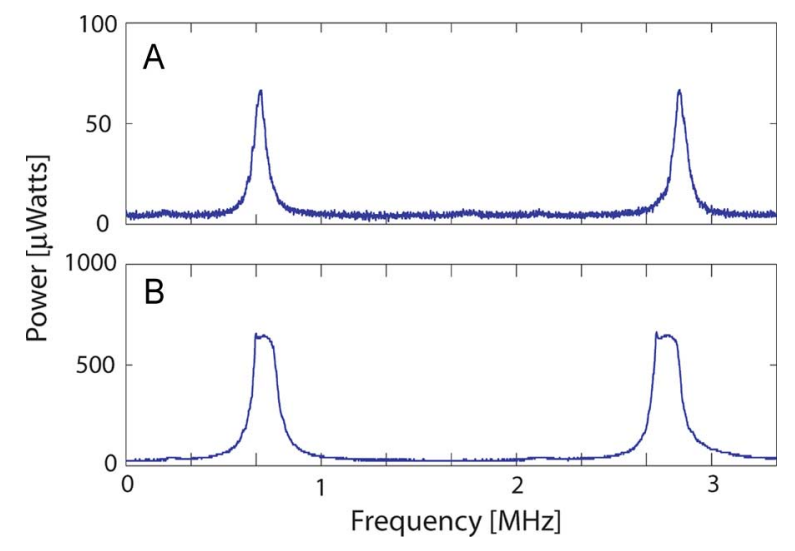

Fig. 2. (A) Transmitted optical power of the FRC as the laser is scanned through several cavity resonances. This is a low-power measurement with a maximum output power of $70 \mu \mathrm{W}$. (B) The same as in trace (A) with high input power so that SBS threshold is exceeded at an output power of approximately $660 \mu \mathrm{W}$.

to a few megahertz [4], and can have little useful effect at frequencies of $50 \mathrm{MHz}$ and above. In addition, such active intensity stabilization systems cannot produce an optical output that is shot-noise-limited [5]. In the high gain limit, the output RIN is the quadrature sum of the shot-noise RIN of the in-loop photodetector and that of the main output. Typically, only a small fraction of the laser output is sampled by the in-loop photodetector, and so the in-loop RIN will dominate and produce an output RIN well above its shot-noise limit.

Hence, there are fundamental difficulties in producing a shot-noise-limited laser source via active stabilization of laser intensity alone. In GW interferometry, a free-space Fabry-Pérot is used to passively low-pass filter the intensity and frequency noise of a solid-state Nd:YAG laser [6]. To achieve the extreme requirements of $\mathrm{GW}$ astronomy, extraordinary measures are taken to isolate these optical cavities from environmental noise, by placing them in vacuum chambers using complex suspension systems to mitigate the effects of seismic, acoustic, mechanical, and thermal noise.

In less demanding industrial applications, such as quantum cryptography, a simpler and more compact solution would suffice to accomplish most of the functions facilitated by a bulk Fabry-Pérot cavity. In this paper, we present a fiber ring cavity (FRC) solution that is effective at low-pass filtering and is simple, small, robust, and cost-effective.

\section{EXPERIMENTAL DEMONSTRATION}

We constructed a passive FRC using $100 \mathrm{~m}$ of single-mode fiber with $13 \mathrm{~dB}$ (or 95\%/5\%) fusion tapered couplers for both input and output couplers. Fig. 2(a) shows the transmitted intensity when the frequency of our DBR fiber laser is scanned through several cavity resonances. This low power plot (maximum power is approximately $70 \mu \mathrm{W}$ ) demonstrates a cavity free-spectral range (FSR) of $2.05 \mathrm{MHz}$, with a finesse of $\sim 32$. Although this finesse is modest, due to the long optical path length of the resonator, the full-width at half-maximum (FWHM) is very narrow at $65 \mathrm{kHz}$, while the resonator has a high $Q$ of $3 \times 10^{9}$.

It is known that Brillion scattering within the FRC scatters light in the reverse direction [7]. The stimulated Brillion scattering (SBS) threshold occurs when the SBS-induced gain at the

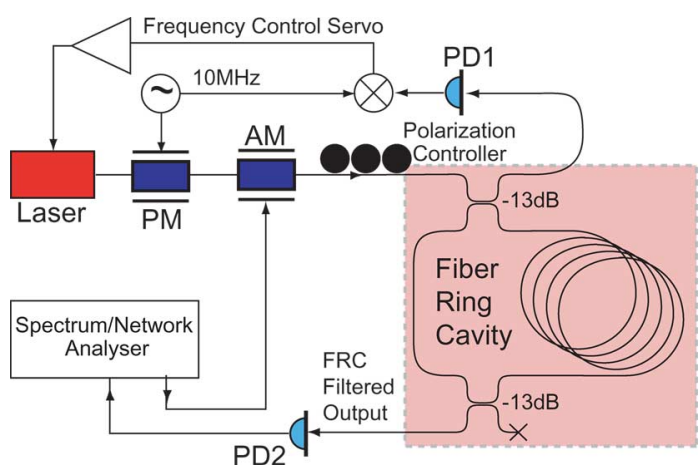

Fig. 3. Simplified schematic of the complete experiment. PM: phase modulator. AM: amplitude modulator. $-13 \mathrm{~dB}$ : fiber fusion tapered coupler with a coupling ratio of $-13 \mathrm{~dB}$. PD1/2: broadband RF photodetectors. $X$ : terminated fiber end.

FRC resonances reaches the cavity single pass loss. Fig. 2(b) shows the transmitted intensity when high power is applied to the cavity input. At approximately $660 \mu \mathrm{W}$, the SBS threshold is reached and the transmitted power is clamped. Below threshold, however, SBS noise amplification still creates large noise peaks at the cavity resonances. In order to eliminate SBS noise on the transmitted output, it was necessary to operate well below the actual SBS threshold. Empirically, we found that operating at an output power of $350 \mu \mathrm{W}$ ensured low noise performance. However, low power operation is not a significant limitation for most quantum optics experiments, where low power filtered seed beams are frequently required [2].

A simplified schematic of the complete experiment is shown in Fig. 3. The DBR fiber laser output is initially phase modulated at $10 \mathrm{MHz}$ before passing through an amplitude modulator. The modulated output is then routed to the FRC input coupler. Five percent of the input light is coupled into the cavity and excites a resonant travelling field within the FRC. After interfering with the cavity field, the input is transmitted through to an RF photodetector, PD1. The detector output is mixed down to create an error signal that is then used to lock the laser output frequency to the FRC resonance. This process is an implementation of the PDH technique [1] applied to an FRC topology.

The narrow optical linewidth of the FRC $(65 \mathrm{kHz})$ places stringent demands on the active control system used to lock the laser frequency to resonance. We utilize a high gain, conditionally stable controller design that minimizes laser frequency excursions from FRC resonance. The controller output is fed to the fiber laser piezoelectric transducer frequency actuator with a unity gain bandwidth of $\approx 4 \mathrm{kHz}$.

To ease the dynamic range requirements of the frequency controller, the FRC is placed within a small anechoic chamber. This chamber dramatically attenuates mechanical and acoustic noise while averaging out ambient temperature fluctuations, thereby passively stabilizing the FRC resonance frequency.

Once the laser is locked to the FRC resonance, it typically stays locked for hours. The output coupler removes $5 \%$ of the intracavity travelling field and directs it via the transmitted output to the ac coupled photodetector PD2. Due to the long storage time of the travelling field inside the FRC (approximately $15 \mu \mathrm{s}$ ), this transmitted output is a time-domain integration of the input field. Consequently, high frequency laser noise is averaged out and removed from the transmitted output. 


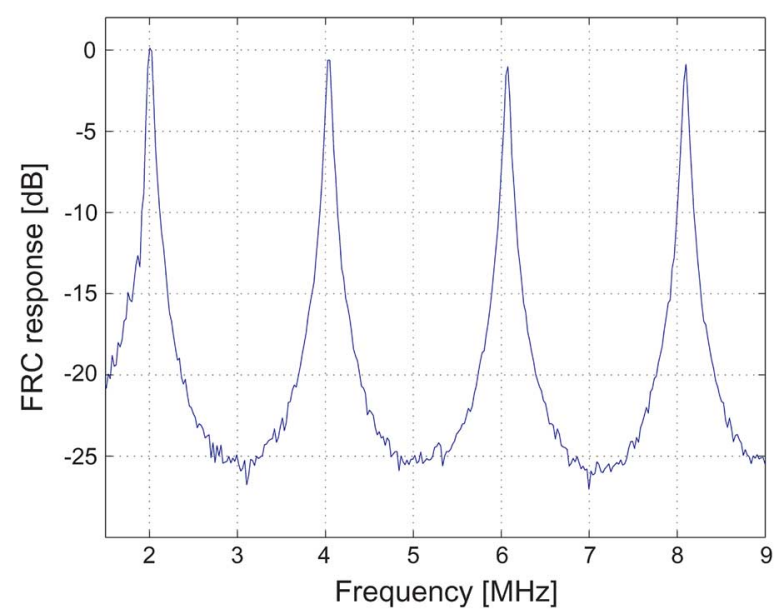

Fig. 4. FRC response to a swept sine input up to $9 \mathrm{MHz}$. The network analyzer output was $+10 \mathrm{dBm}$ into the amplitude modulator. The vertical response is normalized to maximum transmission of the first FSR mode. Note that a significant modulator resonance at approximately $1.5 \mathrm{MHz}$ precluded lower frequency operation.

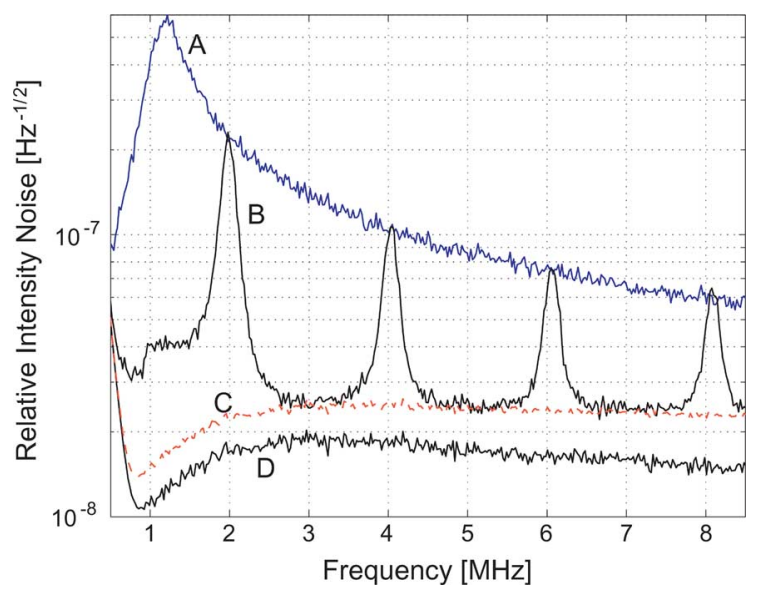

Fig. 5. Filtered intensity noise of the FRC output compared to the intensity noise of the laser at the cavity input. (A) Input intensity noise to the FRC, detected optical power was $350 \mu \mathrm{W}$. (B) Output intensity noise of the FRC, detected optical power was $350 \mu \mathrm{W}$. (C) Sum of calculated shot noise for $350 \mu \mathrm{W}$ and measured electronic noise. (D) Measured electronic noise alone. Both traces (A) and (B) were recorded with the laser RIN control circuit active.

The network analyzer provides a swept sine that is used to drive the amplitude modulator as illustrated in Fig. 3. This creates amplitude modulation (AM) sidebands on the FRC input light field. The carrier and sidebands propagate through the FRC and are detected on PD2. The network analyzer normalizes the PD2 output by the AM drive voltage, yielding the FRC transmission response as a function of frequency [8].

Fig. 4 plots the normalized optical transmission of the FRC from 2 to $9 \mathrm{MHz}$. The FRC attenuates optical sideband features by $26 \mathrm{~dB}$ midway between the Fabry-Pérot resonant modes. In addition, this swept sine response can be used to accurately measure both the FSR and the FWHM of the FRC, verifying the measurements of Fig. 2.

The ability of the FRC filtering to remove laser intensity noise is demonstrated in Fig. 5. Here we compare the RIN of the unfiltered laser (Trace A) with that of the filtered FRC output (Trace
B). The residual laser RO is attenuated by approximately $24 \mathrm{~dB}$ in close agreement with the swept sine measurement of $26 \mathrm{~dB}$ near the midpoint between FSR modes. Noise at the FSR modes is transmitted with no filtering, reaching the unfiltered laser RIN levels at 2.05, 4.1, 6.15, and 8.2 MHz. Trace C plots the sum of the measured electronic noise and the calculated shot noise for $350 \mu \mathrm{W}$ of detected power, taking into account the frequency response of PD2. Trace D plots the equivalent RIN level due to electronic noise alone. Trace B, the filtered laser intensity noise, is seen to coincide with trace $\mathrm{C}$ away from the FSR modes, indicating that over most of the spectrum the filtered laser output does indeed reach the shot-noise limit of this measurement.

The FRC length was designed to be $100 \mathrm{~m}$ in order to ensure that the residual $\mathrm{RO}$ of the laser (at $1.2 \mathrm{MHz}$ as seen in Fig. 5) was approximately antiresonant with the cavity longitudinal modes. This ensures that the suppression of laser intensity noise at the RO frequency is maximized.

We note that the effects of SBS can be reduced by both decreasing the FRC length and its finesse. This allows for enhanced optical throughput at the expense of FRC noise filtering.

The excess laser intensity noise transmitted through the ring resonator at the FSR modes could be removed by cascading a second FRC with an FSR chosen to avoid the FSR modes of the first ring resonator. This would not only remove the remaining technical noise features of the final output, but also attenuate the low frequency noise more efficiently.

\section{CONCLUSION}

We have presented an elegant technique to remove laser intensity noise by locking a laser to a compact and robust FRC using PDH frequency locking. This fiber resonator uses only standard single-mode fiber and fusion tapered couplers. Once locked, the device efficiently removes up to $26 \mathrm{~dB}$ of technical noise and produces an output that is shot-noise-limited over most of the frequency spectrum. Such devices show promise as enabling light sources or transmitters for the industrial application of many quantum optics systems.

\section{REFERENCES}

[1] R. W. P. Drever, J. L. Hall, F. V. Kowalski, J. Hough, G. M. Ford, A. J. Munley, and H. Ward, "Laser phase and frequency stabilization using an optical resonator," Appl. Phys. B, vol. 31, no. 2, pp. 97-105, 1983.

[2] K. McKenzie, N. Grosse, W. P. Bowen, S. E. Whitcomb, M. B. Gray, D E. McClelland, and P. K. Lam, "Squeezing in the audio gravitationalwave detection band," Phys. Rev. Lett., vol. 93, no. 16, p. 161105, 2004.

[3] A. M. Lance, T. Symul, V. Sharma, C. Weedbrook, T. C. Ralph, and P. K. Lam, "No-switching quantum key distribution using broadband modulated coherent light," Phys. Rev. Lett., vol. 95, p. 180503, 2005.

[4] S. Kawamura, A. Abramovici, and M. E. Zucker, "Improved multistage wide band laser frequency stabilization," Rev. Sci. Instrum., vol. 68, pp. 223-229, 1997.

[5] C. C. Harb, M. B. Gray, H.-A. Bachor, R. Schilling, P. Rottengatter, I. Freitag, and H. Welling, "Suppression of the intensity noise in a diode-pumped neodymium:YAG nonplanar ring laser," IEEE $J$. Quantum Electron., vol. 30, no. 12, pp. 2907-2913, Dec. 1994.

[6] B. Willke, N. Uehara, E. K. Gustafson, R. L. Byer, P. King, S. Seel, and R. L. Savage, Jr., "Spatial and temporal filtering of a 10 watt Nd:YAG laser with a ring-cavity, Fabry-Perot, pre-modecleaner," Opt. Lett., vol. 23, no. 21, pp. 1704-1706, 1998.

[7] G. P. Agrawal, Nonlinear Fiber Optics, 2nd ed. San Diego, CA: Academic, 1995

[8] B. J. J. Slagmolen, M. B. Gray, K. G. Baigent, and D. E. McClelland, "Phase-sensitive reflection technique for characterization of a Fabry-Perot interferometer," Appl. Opt., vol. 39, no. 21, pp. 3638-3643, 2000. 